\title{
CONDICIÓN FÍSICA RELACIONADA CON LA SALUD Y SITUACIÓN SOCIOECÓNOMICA DE NIÑOS Y JÓVENES CON DISCAPACIDAD INTELECTUAL DE LOS COLEGIOS DISTRITALES DE LA CIUDAD DE BOGOTÁ
}

\section{Physical condition related to the bealth and socioeconomic situation of children and young people with intellectual disabilities of the district colleges of the City of Bogotá}

Diana Alexandra Camargo Rojas

Facultad de Cultura Física, Deporte y Recreación. Universidad Santo Tomás. Colombia diana.camargo@usantotomas.edu.co

Edwin Arcesio Gómez SERNA

Facultad de Cultura Física, Deporte y Recreación. Universidad Santo Tomás. Colombia

Pablo Sergio Molina Murcia

Facultad de Cultura Física, Deporte y Recreación. Universidad Santo Tomás. Colombia

Recepción: 15 de diciembre de 2017

Aceptación definitiva: 19 de marzo de 2019

Resumen: En el sector educativo sigue siendo un reto para los docentes generar estrategias pedagógicas dentro de sus espacios de clase considerando las características propias de la población. Adicionalmente, los docentes desconocen las condiciones físicas y sociales de sus estudiantes, lo que no permite generar planeaciones acordes a sus necesidades. Objetivo: Caracterizar la condición física y condición socioeconómica de los estudiantes con discapacidad intelectual de los colegios distritales de la ciudad 
CONDICIÓN FÍSICA RELACIONADA CON LA SALUD Y SITUACIÓN SOCIOECÓNOMICA DE NIÑOS Y JÓVENES CON DISCAPACIDAD INTELECTUAL DE LOS COLEGIOS DISTRITALES DE LA CIUDAD DE BOGOTÁ

DIANA ALEXANDRA CAMARGO ROJAS, EDWIN ARCESIO GÓMEZ SERNA Y PABLO SERGIO MOLINA MURCIA

de Bogotá para población con discapacidad. Método: Estudio de corte transversal, que empleó baterías como la Brockport y la encuesta STEPS de la OMS. Población: 132 niños y jóvenes con discapacidad intelectual. Resultados: En cuanto a la composición corporal, el $62.12 \%$ se encuentra en la zona saludable y el $6.82 \%$ presenta bajo peso, lo cual requiere seguimiento por profesionales de nutrición; así mismo el $17.42 \%$ presenta alto riesgo, población que requiere seguimiento. En relación con la condición física la mayoría de la población, un $72.06 \%$, necesita mejorar en la fuerza prensil y la fuerza de abdominales, variables relacionadas con el riesgo cardiovascular. Por último, es importante mencionar que el $73 \%$ de la población se encuentra en los estratos 1 y 2, lo cual puede influir en la práctica de actividad física. Conclusión: La población tiene una condición física baja que se asocia como en otros estudios con el estado socioeconómico de las familias. El riesgo cardiovascular expresado en porcentaje de grasa continúa siendo un elemento para trabajar en la población.

Palabras clave: personas con discapacidad; aptitud física; actividad física; clase social; educación.

АвSTRACт: In the education sector is a challenge for teachers to generate pedagogical strategies within their classroom considering the characteristics of the population. Additionally, teachers do not know the physical and social conditions of their students, which they can't generate plans according to their needs. Objective: To characterize the physical condition and socioeconomic status of students with intellectual disabilities in the district schools of the city of Bogotá for people with disabilities. Method: Crosssectional study, which used Brockport physical fitness test and the WHO STEPS survey. Population: 132 children and young people with intellectual disabilities. Results: In terms of body composition, $62.12 \%$ are in the healthy area and $6.82 \%$ have low weight, which requires follow-up by nutrition professionals; and $17.42 \%$ present high risk, a population that requires follow-up. In relation to the physical fitness the majority of the population, a $72.06 \%$, need to improve in the grip strength and strength of abdominals, variables related to cardiovascular risk. Finally, it is important to mention that $73 \%$ of the population is in stratum 1 and 2, which can influence the practice of physical activity. Conclusion: The population has a low physical fitness that is associated as in other studies with the socioeconomic status of families. The cardiovascular risk expressed in percentage of fat continues to be an element to work in the population.

KEY WORDs: disabled person; physical fitness; exercise; social class; education.

\section{Introducción}

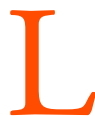

A INCLUSIÓn EDUCATIVA, ámbito en el cual se desarrolló la presente investigación, en nuestro país ha sido preocupación del Estado. A través del Ministerio de Educación Nacional se establecieron los parámetros y criterios que orientan la prestación del servicio educativo para la población que presenta discapacidad física, psíquica, sensorial, cognitiva, emocional. Como lo plantea Villarreal (2011), este proceso no ha sido una tarea fácil, pues "uno de los ejes en torno al cual se han venido

(C) Ediciones Universidad de Salamanca / CC BY-NC-ND

Siglo Cero, vol. 50 (4), n. ${ }^{\circ}$ 272, 2019, octubre-diciembre, pp. 39-59 
CONDICIÓN FÍSICA RELACIONADA CON LA SALUD Y SITUACIÓN SOCIOECÓNOMICA DE NIÑOS Y JÓVENES CON DISCAPACIDAD INTELECTUAL DE LOS COLEGIOS DISTRITALES DE LA CIUDAD DE BOGOTÁ

DIANA ALEXANDRA CAMARGO ROJAS, EDWIN ARCESIO GÓMEZ SERNA Y PABLO SERGIO MOLINA MURCIA

desenvolviendo las políticas educativas en Colombia es la cobertura, la que tiene como objetivo que la totalidad de los niños y jóvenes en edad escolar ingresen a las instituciones de educación básica y media. Este aumento de la cobertura ha traído consigo algunos problemas, debido a la diversidad de los estudiantes que han ingresado a las aulas y que han planteado nuevos retos a la labor educativa" (p. 1).

Con relación a lo anterior, el Ministerio de Educación Nacional (MEN) (2007) afirma:

Los datos del Censo de 2005 reportan 392.084 menores de 18 años con discapacidad, de los cuales 270.593 asisten a la escuela y 119,831 no lo hacen. Desde 2003 y hasta 2006, las secretarías reportan la matrícula de 81.757 estudiantes con discapacidad en 4.369 establecimientos educativos. A pesar de estos avances, indudablemente significativos, aún son grandes los retos para lograr que todos ingresen al sistema educativo y sean educados con pertinencia y calidad (p. 1).

Cabe aclarar que el reporte dado por el MEN no presenta datos diferenciales entre los establecimientos educativos incluyentes y los denominados de aula excepcional, los datos los presenta de manera consolidada.

Otra de las problemáticas con las que se encuentra la escuela es la presencia de enfermedades no transmisibles (ENT) en la población escolar, atribuidas a la inactividad física. Esta situación evidencia que, en un escenario cultural tan globalizado como en el que vivimos actualmente, la sofisticación tecnológica ha venido a pasos agigantados ocupando el puesto dedicado por el ser humano al movimiento de su cuerpo, dando paso al sedentarismo que no distingue en sus consecuencias ni raza, ni sexo, ni condición social, pues el actual ser humano se caracteriza por sus bajos niveles de actividad física, un alto consumo de alimentos refinados hipercalóricos y el consumo también de tabaco y alcohol. Buhrigh et al. (2009) afirman que el sedentarismo ha tenido un incremento sostenido a nivel mundial, indistintamente del género, edad y situación socioeconómica, lo que se traduce en un problema emergente tanto de manera local como global, que afecta a niños, niñas y adolescentes con y sin discapacidad.

Según Vidarte, Vélez y Parra (2012), "La inactividad constituye uno de los grandes factores de riesgo que explican las proporciones epidémicas actuales de las enfermedades no transmisibles (ENT)" (p. 422). Esta situación se corroboró cuando la American Heart Association (AHA) (2007, citada por Vidarte, Vélez y Parra, 2012), basada en evidencias científicas, anunció: "La vida sedentaria fue oficialmente ascendida a factor de riesgo mayor e independiente de enfermedad coronaria” (p. 422); lo que convierte a la población con Discapacidad Intelectual (DI) en blanco de las enfermedades causadas por un estilo de vida poco activo y alejado de la actividad física.

Según algunos estudios relacionados a continuación la inactividad física se incrementa de forma alarmante en niños y jóvenes con DI ya que los niveles de actividad física de estos se encuentran por debajo de lo normal, al compararlos con sus pares sin discapacidad (Sherrill, 1993; Moreno, 2001; citados por Ortiz, 2010). A pesar de que este grupo poblacional se considera en riesgo y es consumidor frecuente de los

(C) Ediciones Universidad de Salamanca / CC BY-NC-ND

Siglo Cero, vol. 50 (4), n. ${ }^{\circ}$ 272, 2019, octubre-diciembre, pp. 39-59 
CONDICIÓN FÍSICA RELACIONADA CON LA SALUD Y SITUACIÓN SOCIOECÓNOMICA DE NIÑOS Y JÓVENES CON DISCAPACIDAD INTELECTUAL DE LOS COLEGIOS DISTRITALES DE LA CIUDAD DE BOGOTÁ

DIANA ALEXANDRA CAMARGO ROJAS, EDWIN ARCESIO GÓMEZ SERNA Y PABLO SERGIO MOLINA MURCIA

servicios de salud a nivel mundial, no se encuentran estudios nacionales a este respecto (Cossío y Bolaños, 2015).

A nivel internacional encontramos estudios como el de Ortiz (2010), quien plantea que las personas con discapacidad intelectual tienen bajos niveles de actividad física (AF). Igualmente, Foley, Harvey, Hae-j y So-Yeum (2008) encontraron en su estudio que los estudiantes con DI tienden a tener altos índices de obesidad y menos desarrollo de sus destrezas motrices. Kreb (2000) indicó que las personas con DI tienden a ser menos activos, reflejan bajos niveles de aptitud física y desarrollo motriz y su vida cotidiana es sedentaria. Ayyangar (2002) (citado por Ortiz, 2010) encontró que los niños con DI están particularmente en riesgo debido a la falta de participación en la actividad física. Finalmente, Kleinert et al. (2007) indican que otro obstáculo riesgoso en los adolescentes con DI es el mal uso del tiempo libre.

Sobre la base de las consideraciones anteriores, es evidente que las poblaciones con DI, al igual que otras discapacidades, se ven afectadas al igual que la población sin discapacidad por las enfermedades no transmisibles, lo que se agudiza en esta población por la falta de acceso a una actividad física dirigida y adaptada a sus condiciones, reconociendo sus particularidades, capacidades y necesidades.

Es preciso, señalar entonces, que la situación local no difiere de la situación planteada pues a nivel distrital esta problemática cobra más relevancia, dado que la población objeto sufre el impacto de vivir en una ciudad compleja donde factores sociodemográficos, culturales, ambientales y de educación siguen generando discriminación hacia la población con DI y las barreras de acceso son mayores. Otros factores asociados con el tiempo libre y las actividades dedicadas a este por los jóvenes con DI, son, por ejemplo, el gran número de horas que dedican los jóvenes a ver televisión, junto con el tiempo dedicado a las redes sociales, en contraposición con el poco tiempo dedicado al ejercicio físico, lo cual puede darse por la poca accesibilidad a escenarios seguros para estas prácticas y a la falta de conocimiento sobre la oferta que la ciudad ofrece, por parte de familiares y cuidadores.

Otros factores que influyen en el aumento del sedentarismo de los jóvenes con DI, tienen que ver con el poco apoyo que reciben las familias para generar y participar en actividades extraescolares. Bogotá es muy insegura y presenta una movilidad compleja, pues las distancias son muy largas como para que estos jóvenes se desplacen solos a participar de eventos relacionados con la actividad física, la cultura y la recreación que ofrecen las distintas organizaciones sociales de la ciudad (Moreno, 2007).

A estos factores se le pueden sumar de igual manera los generados en el ámbito escolar por parte de aquellos docentes que dejan sin su clase de educación física a los jóvenes, como parte de un castigo e incluso no permiten que jueguen en el recreo. Al ejemplo anterior se le puede sumar la discriminación entre estudiantes, a quienes de igual manera no les gusta jugar con niños con obesidad y mucho menos con discapacidad, provocando en muchos casos la aversión hacia la clase de educación física, caso que es más evidente en las niñas.

Bogotá, ciudad en la cual se desarrolla el presente estudio, todavía no tiene la suficiente sensibilización e información real sobre las características de las personas con

(C) Ediciones Universidad de Salamanca / CC BY-NC-ND

Siglo Cero, vol. 50 (4), n. ${ }^{\circ}$ 272, 2019, octubre-diciembre, pp. 39-59 
CONDICIÓN FÍSICA RELACIONADA CON LA SALUD Y SITUACIÓN SOCIOECÓNOMICA DE NIÑOS Y JÓVENES CON DISCAPACIDAD INTELECTUAL DE LOS COLEGIOS DISTRITALES DE LA CIUDAD DE BOGOTÁ

DIANA ALEXANDRA CAMARGO ROJAS, EDWIN ARCESIO GÓMEZ SERNA Y PABLO SERGIO MOLINA MURCIA

DI y tiene imaginarios falsos y perjudiciales que se traducen en actitudes negativas que impiden los debidos procesos de inclusión educativa y social (Moreno, 2007).

En definitiva, conocer y reconocer la condición física, relacionada con la salud, de los escolares con DI puede llevar a replantear los programas curriculares de la educación física de los colegios del Distrito capital, creando consciencia sobre la importancia de incorporar el ejercicio físico como parte fundamental de las denominadas actividades de la vida diaria (AVD) de estos sujetos; pues la actividad física se ha considerado como un coadyuvante de los estilos de vida saludables en la prevención de enfermedades, favoreciendo en gran medida la interacción y las relaciones sociales tan escasas en esta población. Este mismo conocimiento pondrá en alerta y sobre aviso a las directivas del sistema educativo distrital, para que vuelvan su mirada a las poblaciones con DI, las cuales necesitan más actividad física, e incentivar en ellos y sus familias estilos de vida más saludables, a través de políticas que formalicen programas en pro de estas iniciativas.

La valoración de diferentes manifestaciones de la condición física relacionada con la salud, a través de pruebas físicas y antropométricas de campo, fiables y avaladas internacionalmente, permitirá establecer un diagnóstico preciso sobre los factores de riesgo que estén presentes en los jóvenes con DI con la intención pedagógica de generar estrategias de promoción de la salud y prevención de las ENT, ya que estos estudios con dicha población son escasos.

Siguiendo esta línea de responsabilidad institucional, la educación física es la llamada desde sus elementos constitutivos (juegos, deportes, gimnasia, danza, etc.) a desarrollar cualidades deseables, tales como habilidades sociales, conocimiento moral, disciplina y salud (Contreras, 2009), generando desde su puesta en práctica en la escuela la tendencia con énfasis en la estética y la salud, cuyo objeto fundamental es la creación de hábitos de actividad física en los alumnos (Contreras, 2009).

Con el fin de desarrollar el presente estudio es necesario revisar algunos antecedentes relacionados con el tema, los cuales se han desarrollado a lo largo de los últimos años y permiten comprender la problemática aquí abordada.

Según Shin y Park (2012), las personas con DI tienen bajos niveles de resistencia cardiovascular y fuerza muscular, así como altos niveles de obesidad en comparación de pares sin discapacidad. Igualmente, las personas con DI tienen sustancialmente menor rendimiento de aptitud física que la población general (Frey, Stanish y Temple, 2008; Montgomery, Reid y Koziris, 1992; Pitetti y Campbell, 1991; citados por Shin y Park, 2012) y altas de obesidad, como lo plantean los estudios de Choi et al. en el 2012, Frey y Chow en el 2006 y Lin et al. en el 2005 (citados por Li, Frey, McCormick y Johnston, 2015).

Por otro lado, Li, Frey, McCormick y Johnston (2015), en su estudio, encuentran, basados en estándares específicos por país, que los atletas con DI estadounidenses son más obesos que los atletas con DI de China $(\mathrm{OR}=2.47, \mathrm{p}<0.001)$. Igualmente, encontraron que al incrementar la edad incrementaron los odds por obesidad para un factor de 1.04, y que los odds de ocurrencia de obesidad en los atletas estadounidenses fueron 2.47 veces mayores que los de los atletas de China. Lo cual evidencia asociaciones entre países y la edad con la ocurrencia de obesidad en atletas con DI. 
CONDICIÓN FÍSICA RELACIONADA CON LA SALUD Y SITUACIÓN SOCIOECÓNOMICA DE NIÑOS Y JÓVENES CON DISCAPACIDAD INTELECTUAL DE LOS COLEGIOS DISTRITALES DE LA CIUDAD DE BOGOTÁ

DIANA ALEXANDRA CAMARGO ROJAS, EDWIN ARCESIO GÓMEZ SERNA Y PABLO SERGIO MOLINA MURCIA

En Barcelona, España, Bofill (2008) elabora una tesis doctoral titulada: Valoración de la Condición Física en la Discapacidad Intelectual, que surge de la necesidad como profesional de la educación física que trabaja con personas con discapacidad intelectual de poder evaluar la condición física sin necesidad de realizar complejas pruebas de laboratorio o requerir la ayuda de otros profesionales. Se utilizaron diferentes protocolos para la administración de las siguientes pruebas: velocidad $20 \mathrm{~m}$, dinamometría de manos, dinamometría de piernas, potencia aeróbica máxima. La muestra de este estudio estuvo constituida por 46 individuos, 31 hombres y 15 mujeres, con diferentes tipos de discapacidad intelectual.

Otro estudio realizado en la ciudad de Puerto Rico, por Ortiz (2010), tuvo como propósito analizar el estado de la situación de la aptitud física en niños, niñas y jóvenes con discapacidad intelectual entre las edades escolares de 6 a 18 años. La investigadora preparó una plantilla de comparación analítica de estudios en aptitud física de escolares con discapacidad intelectual. La comparación analítica de este estudio se usó para determinar los niveles de las destrezas motrices y eficiencia física según su nivel de discapacidad intelectual. A partir de esto, se observó que los que tienen discapacidad leve poseen mejores promedios que los diagnosticados con discapacidad intelectual severa. En cuanto a los estudiantes con discapacidad intelectual moderada, los resultados variaron ya que, en diferentes reactivos, sus resultados arrojaron igualdad o menos rendimiento que los niños y niñas con discapacidad intelectual leve (Ortiz, 2010).

Este recorrido académico y social permitió plantear como pregunta de investigación: ¿ cuál es la condición física y la condición socioeconómica de los estudiantes con DI de los colegios distritales de la ciudad de Bogotá? La respuesta permitiría dar inicio a programas de actividad física incluyentes que consideren la diferencia sin generar discriminación.

\section{Método}

El presente estudio es una investigación con paradigma positivista y enfoque cuantitativo: diseño de investigación analítico de corte transversal. Se desarrolló en 132 estudiantes con discapacidad intelectual entre los 10 a los 17 años de colegios distritales para población con discapacidad y condiciones excepcionales (en el caso del sistema educativo de Bogotá, Colombia, los colegios para la población con discapacidad son exclusivos para esta población, por lo tanto, pueden ser considerados de educación especial), que cumplieron con los criterios de inclusión: ser estudiantes con DI entre los 10 a los 17 años cuyos padres y/o cuidadores hayan firmado el consentimiento informado; población diagnosticada por medio del diagnóstico médico basado en el CIE 10, con retraso mental leve (F70) y retraso mental moderado (F71); no contar con restricción médica para la práctica de la actividad física o que tengan algún impedimento físico que no les permita realizar las pruebas como una alteración neuromuscular, comorbilidades cardiometabólicas y osteomuscular severa.

(C) Ediciones Universidad de Salamanca / CC BY-NC-ND

Siglo Cero, vol. 50 (4), n. ${ }^{\circ}$ 272, 2019, octubre-diciembre, pp. 39-59 


\subsection{Variables de estudio}

El Cuadro 1 recopila las variables estudiadas, su definición, su escala de medición y el instrumento a utilizar.

\begin{tabular}{|c|c|c|c|}
\hline \multicolumn{4}{|c|}{ Cuadro 1} \\
\hline VARIABLES & DEFinición & ESCALA O DIMENSIÓN & INSTRUMENTO \\
\hline 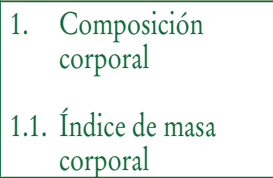 & $\begin{array}{l}\text { Es un indicador simple de la relación } \\
\text { entre el peso y la talla, que se utiliza } \\
\text { para identificar sobrepeso y obesi- } \\
\text { dad. Se calcula dividiendo talla por el } \\
\text { peso al cuadrado. }\end{array}$ & Estándares Brockport. & $\begin{array}{l}\text { Tallímetro } \\
\text { Báscula }\end{array}$ \\
\hline 1.2. Porcentaje de grasa & $\begin{array}{l}\text { Es un método que a partir de la de- } \\
\text { terminación del espesor del tejido } \\
\text { adiposo subcutáneo permite evaluar } \\
\text { las características de la composición } \\
\text { corporal con respecto a la calidad } \\
\text { relativa del peso y masa grasa de la } \\
\text { persona. Fernández y Hoyos (2007). }\end{array}$ & $\begin{array}{l}\text { Suma de } 3 \text { pliegues: tríceps, sub } \\
\text { escapular y pantorrilla (The } \\
\text { Brockport). }\end{array}$ & $\begin{array}{l}\text { Adipómetro } \\
\text { Impedancia }\end{array}$ \\
\hline 2. Flexibilidad & \multicolumn{3}{|c|}{$\begin{array}{l}\text { Esta cualidad física permite un máximo recorrido de las articulaciones gracias a la elas- } \\
\text { ticidad y extensibilidad de los músculos que se insertan alrededor de cada una de ellas } \\
\text { (Fernández y Hoyos, 2007). }\end{array}$} \\
\hline $\begin{array}{l}\text { 2.1. Flexibilidad } \\
\text { componente } \\
\text { posterior de } \\
\text { tronco y miembros } \\
\text { inferiores } \\
\end{array}$ & $\begin{array}{l}\text { Flexión profunda del tronco. Valora } \\
\text { la flexibilidad de los músculos poste- } \\
\text { riores antigravitorios isquiotibiales y } \\
\text { zona lumbar. }\end{array}$ & $\begin{array}{l}\text { Escala para clasificar los resul- } \\
\text { tados del sit and reach modifi- } \\
\text { cado según Bateria Brockport. }\end{array}$ & $\begin{array}{l}\text { Cajón demarcado } \\
\text { en centímetros }\end{array}$ \\
\hline \begin{tabular}{|l|} 
2.2. Rotación de \\
hombros. Bilateral
\end{tabular} & $\begin{array}{l}\text { Mide la flexibilidad de los músculos } \\
\text { del hombro y cintura escapular. }\end{array}$ & $\begin{array}{l}1 \text { a } 3 \text { de acuerdo a Batería de Broc- } \\
\text { kport (Winnick y Short, 1999). }\end{array}$ & \\
\hline \begin{tabular}{|ll} 
3. & Fuerza de \\
& resistencia \\
\end{tabular} & \multicolumn{3}{|c|}{$\begin{array}{l}\text { Es la capacidad que nos permite, mediante acciones musculares llamadas contracciones, } \\
\text { vencer una resistencia u oponerse a ella. }\end{array}$} \\
\hline $\begin{array}{l}\text { 3.1. Fuerza de } \\
\text { abdominales }\end{array}$ & $\begin{array}{l}\text { Evalúa la resistencia de los músculos } \\
\text { abdominales. }\end{array}$ & $\begin{array}{l}\text { Se cuentan cuantas repeticiones } \\
\text { sea posible con una cadencia es- } \\
\text { tablecida (CD) de } 20 \text { abd. x mi- } \\
\text { nuto (Winnick y Short, 1999). } \\
\end{array}$ & $\begin{array}{l}\text { Colchoneta } \\
\text { Metrónomo }\end{array}$ \\
\hline 3.2. Fuerza prensil & $\begin{array}{l}\text { Mide la fuerza de apretón de cada mano } \\
\text { a partir del área muscular braquial. }\end{array}$ & $\begin{array}{l}\text { Escala de evaluación propuesta } \\
\text { por Batería Brockport (Win- } \\
\text { nick y Short, 1999). }\end{array}$ & Dinamómetro \\
\hline \multirow[t]{2}{*}{ 4. Potencia aeróbica } & \multicolumn{3}{|c|}{$\begin{array}{l}\text { Se trata de un componente importante de la condición física ya que como se indica en la } \\
\text { literatura refleja la capacidad global de los sistemas cardiovascular y respiratorio; así como } \\
\text { la capacidad de llevar a cabo esfuerzos prolongados. }\end{array}$} \\
\hline & $\begin{array}{l}\text { Es un test indirecto para la valoración } \\
\text { en campo de la capacidad aeróbica } \\
\text { mediante una carrera de ida y vuelta } \\
\text { entre dos líneas paralelas separadas } \\
\text { por } 20 \mathrm{~m} \text { (Winnick y Short, 1999). }\end{array}$ & $\begin{array}{l}\text { Ecuación de Leger: } \\
\text { VO2Max }=31.025+(3.238 \mathrm{xVel}) \\
-(3.248 \mathrm{xedad})+(0.1536 \mathrm{xVel} \mathrm{x} \\
\text { edad). } \\
\text { Escala de evaluación propuesta } \\
\text { por Batería Brockport (Win- } \\
\text { nick y Short, 1999). }\end{array}$ & $\begin{array}{l}\text { Test de } 20 \mathrm{~m} \text { ida } \\
\text { y vuelta. PACER }\end{array}$ \\
\hline
\end{tabular}

() Ediciones Universidad de Salamanca / CC BY-NC-ND

Siglo Cero, vol. 50 (4), n. ${ }^{\circ} 272,2019$, octubre-diciembre, pp. 39-59 


\begin{tabular}{|c|c|c|c|}
\hline \multicolumn{4}{|c|}{ CUADRO 1 (cont.) } \\
\hline VARIABLES & Definición & ESCALA O DIMENSIÓN & INSTRUMENTO \\
\hline \begin{tabular}{|ll}
5. & Condición \\
& socioeconómica- \\
& clase social
\end{tabular} & $\begin{array}{l}\text { Condición social que abarca múlti- } \\
\text { ples factores como el nivel de ingre- } \\
\text { sos, el nivel académico y el estatus } \\
\text { social. }\end{array}$ & $\begin{array}{l}\text { Escalas cualitativas de ingreso } \\
\text { mensual, estrato, vivienda, ocu- } \\
\text { pación, nivel educativo, entre } \\
\text { otros. El estrato socioeconómi- } \\
\text { co para la población colombia- } \\
\text { na está definido las condiciones } \\
\text { sociales, acceso a servicios pú- } \\
\text { blicos y poder adquisitivo de } \\
\text { las localidades, comunas y ba- } \\
\text { rios de las ciudades. }\end{array}$ & $\begin{array}{l}\text { Encuesta STEPS } \\
\text { OMS }\end{array}$ \\
\hline
\end{tabular}

A continuación, se conceptualizan algunas variables e instrumentos que fueron empleados en el presente estudio.

\subsubsection{Discapacidad intelectual}

Para el propósito de este proyecto, intervendrán únicamente estudiantes con DI, por ser una de las poblaciones más afectada dentro de los procesos inclusivos y por poseer altos índices de obesidad, y con ello riesgo para adquirir una ECNT (Foley, Hervey, Hae-ja y So-Yeum, 2008; Sherill, 1993; Moreno, 2001, citados por Ortiz, 2010).

Según la American Association on Intellectual and Developmental Disabilities (AAIDD) (2010), la discapacidad intelectual es:

una discapacidad caracterizada por limitaciones significativas tanto en el funcionamiento intelectual (razonamiento, aprendizaje, resolución de problemas) como en el comportamiento adaptativo, que abarca una gama de habilidades sociales y prácticas cotidianas. Esta discapacidad se origina antes de los 18 años (p. 1).

La definición de la población en el presente estudio se realizó por medio del diagnóstico médico que es entregado en la institución educativa, el cual define según el CIE-10 el nivel de discapacidad.

\subsubsection{La clase social: condición o posición socioeconómica}

Según Krieger (2002) “la posición socioeconómica es un concepto agregado que incluye medidas basadas tanto en los recursos como en el prestigio, relacionadas con la posición de clase social en la infancia y en la edad adulta. Las medidas basadas en los recursos se refieren a los recursos y activos materiales y sociales, entre ellos los ingresos, la riqueza y las credenciales educacionales” (p. 486). Por otro lado, el término de clase social traído de teorías sociológicas importantes como la de Marx y Weber "se refiere a grupos sociales surgidos de las relaciones económicas interdependientes 
CONDICIÓN FÍSICA RELACIONADA CON LA SALUD Y SITUACIÓN SOCIOECÓNOMICA DE NIÑOS Y JÓVENES CON DISCAPACIDAD INTELECTUAL DE LOS COLEGIOS DISTRITALES DE LA CIUDAD DE BOGOTÁ DIANA ALEXANDRA CAMARGO ROJAS, EDWIN ARCESIO GÓMEZ SERNA Y PABLO SERGIO MOLINA MURCIA

entre las personas” (Krieger, 2002: 486). Esta condición social que abarca múltiples factores, como el nivel de ingresos, el nivel académico y el estatus social, nos acerca a la comprensión de las dinámicas en las que confluye la población y cómo estas pueden llegar a determinar unas formas de concebir y llevar a cabo las prácticas de actividad física en un contexto social específico. Para el presente estudio se empleará un apartado de la Encuesta STEP de la OMS.

\subsubsection{Condición física}

Para Torres (1996) (citado por Casimiro, 2002) la condición física es “el conjunto de condiciones anatómicas, fisiológicas y motoras, que hacen falta para la realización de esfuerzos físicos y musculares, tanto en el trabajo como en el deporte” (p. 18). Por otro lado, el ACSM (American College of Sports Medicine) define Physical fitness o aptitud física como un set de atributos o características individuales relacionados con la habilidad para desempeñar una actividad física; estas características usualmente están relacionadas con la salud y con el desarrollo de destrezas motrices (American College of Sports Medicine, 2014).

2.1.4. Evaluación de la condición física en personas con discapacidad - Batería de Brockport

La prueba de Aptitud Física de Brockport (BPFT) (Winnick y Short, 1999) es una prueba referida a criterios relacionados con la salud. La BPFT está diseñada y validada principalmente para su uso entre los jóvenes con discapacidad física e intelectual. Sigue un proceso de tres pasos:

- Precisión clasificación o sub-clasificación de cada joven.

- Selección los elementos de prueba pertinentes.

- Administración de los elementos de prueba elegido para medir el estado de forma física.

La primera versión del Brockport test manual fue publicada en 1999 por la editorial Human Kinetics. En esta ya se encontraron los criterios de evaluación y clasificación de las diferentes cualidades que influyen sobre la aptitud física, se establecen los requerimientos mínimos para la aplicación del test y todo el contenido epistemológico y conceptual con el cual se desarrolló la batería de evaluación. Winnick y Short (2014) publican nuevamente de la mano de Human Kinetics la segunda versión de la batería, la cual presenta mínimas diferencias comparada con la primera, pero cuenta con los estudios de validez y confiabilidad realizados por los autores en el 2005.

\subsection{Plan de análisis}

Los datos fueron registrados en una base de datos de Excel, la cual se exportó al programa SPSS versión 20.1, con el fin de realizar los análisis univariados y bivariados

(C) Ediciones Universidad de Salamanca / CC BY-NC-ND

Siglo Cero, vol. 50 (4), n. ${ }^{\circ}$ 272, 2019, octubre-diciembre, pp. 39-59 
CONDICIÓN FÍSICA RELACIONADA CON LA SALUD Y SITUACIÓN SOCIOECÓNOMICA DE NIÑOS Y JÓVENES CON DISCAPACIDAD INTELECTUAL DE LOS COLEGIOS DISTRITALES DE LA CIUDAD DE BOGOTÁ

DIANA ALEXANDRA CAMARGO ROJAS, EDWIN ARCESIO GÓMEZ SERNA Y PABLO SERGIO MOLINA MURCIA

respectivos, a través de la estadística descriptiva y analítica. Para aquellas variables continuas con comportamiento normal se aplicaron medidas de tendencia central tales como: media y desviación estándar con estadísticos de prueba: T de Student para diferencias de medias en dos grupos; en el caso de la variable discapacidad que tiene más de dos grupos se realizará una ANOVA para la signficancia entre grupos. Para las variables no paramétricas se utiliza mediana con rango intercuartílico con la prueba no paramétrica U Mann Whitney. Para variables categóricas se utilizaron frecuencias absolutas, proporciones y tablas de contingencia.

\subsection{Consideraciones éticas}

La presente investigación considera los postulados de la Declaración de Helsinki y la Resolución 8430 de 1993 relacionada con investigaciones clínicas en Colombia, siendo una investigación de riesgo moderado por realizar intervenciones que pueden cambiar las condiciones fisiológicas de base del sujeto. Por lo anterior, no se desarrollará en población con contraindicación en la práctica de actividad física o con alguna patología de base que pueda verse afectada con la realización de las pruebas. Igualmente, considerando la Resolución 8430 se aplicará un consentimiento informado a los padres de familias, quienes autorizan o no la participación de sus hijos. Este proyecto fue aprobado por el Consejo de Facultad de la Facultad de Cultura Física, Deporte y Recreación, así como por la Unidad de Investigación de la Universidad Santo Tomás.

\section{Resultados}

Contando con un total de 175 niños y jóvenes en el Colegio Gustavo Restrepo y 49 niños y jóvenes del Colegio las Américas, finalmente cumplieron con todos los requisitos 132 niños y jóvenes con discapacidad intelectual.

En la Tabla 1 se describen las medidas de tendencia central de las variables de estudio en la población general, observando que la media del IMC se encuentra en 21.90, lo que representa peso normal en la mayoría de la población; no obstante, al encontrar un límite inferior en 14.03 y un límite superior en 32.31, es importante tener en cuenta estos datos, reconociendo a la población que puede estar en alguna condición de riesgo. Por otro lado, con relación a la sumatoria de pliegues, un elemento a tener en cuenta desde la OMS, se evidencia una media de 40.03 en la población evaluada, lo que representa un riesgo alto para la población, y puede llegar ser un factor de riesgo cardiovascular.

\begin{tabular}{|l|c|c|c|c|c|}
\hline \multicolumn{7}{|c|}{ TABLA 1. Estadísticos descriptivos } \\
\hline \multicolumn{1}{|c|}{ VARIABLES } & $\mathrm{N}$ & MínImo & Máximo & MEDIA & DEsv. Típ. \\
\hline EDAD & 132 & 10.00 & 17.00 & 15.1439 & 1.76104 \\
\hline IMC & 120 & 14.03 & 32.31 & 21.9073 & 3.74102 \\
\hline SUMPLIEGUES & 65 & 12.00 & 69.00 & 40.0338 & 11.14476 \\
\hline PORCGRASA & 77 & 5.00 & 44.90 & 21.4662 & 8.91301 \\
\hline
\end{tabular}

(C) Ediciones Universidad de Salamanca / CC BY-NC-ND

Siglo Cero, vol. 50 (4), n. ${ }^{\circ}$ 272, 2019, octubre-diciembre, pp. 39-59 
CONDICIÓN FÍSICA RELACIONADA CON LA SALUD Y SITUACIÓN SOCIOECÓNOMICA DE NIÑOS Y JÓVENES CON DISCAPACIDAD INTELECTUAL DE LOS COLEGIOS DISTRITALES DE LA CIUDAD DE BOGOTÁ DIANA ALEXANDRA CAMARGO ROJAS, EDWIN ARCESIO GÓMEZ SERNA Y PABLO SERGIO MOLINA MURCIA

TABLA 1. Estadísticos descriptivos (cont.)

\begin{tabular}{|l|r|r|r|r|r|}
\hline \multicolumn{1}{|c|}{ VARIABLES } & \multicolumn{1}{c|}{$\mathrm{N}$} & \multicolumn{1}{c|}{ MÍNIMO } & \multicolumn{1}{c|}{ MáxImo } & \multicolumn{1}{c|}{ MEdia } & \multicolumn{1}{c|}{ Desv. TíP. } \\
\hline FPRENSIL & 118 & .00 & 48.00 & 19.0169 & 7.94638 \\
\hline SITANDREACH & 114 & -13.00 & 37.00 & 13.5000 & 10.21126 \\
\hline CURLUP & 123 & .00 & 46.00 & 14.9593 & 12.52695 \\
\hline TRUNKLIFT & 58 & 5.00 & 48.00 & 20.1345 & 8.55737 \\
\hline PACER & 75 & 3.00 & 106.00 & 28.0400 & 22.27375 \\
\hline
\end{tabular}

Elaboración propia, 2017.

En cuanto a las variables relacionadas con la condición física, se evidencia una condición baja en las cualidades de fuerza muscular y capacidad aeróbica; no obstante, las desviaciones estándar son altas, por lo que es necesario revisar los casos que particularmente obtuvieron mejores resultados.

En cuanto al tipo de discapacidad, la mayoría de la población fue diagnosticada con retraso mental leve, condición que prevalece en los colegios, por favorecer los procesos de inclusión educativa. Solo el $13.38 \%$ presenta síndrome de Down, otra condición que se incluye dentro de la DI, pero que por sus características neurofisiológicas puede presentar diferencias en la condición física para la salud (ver Tabla 2).

\begin{tabular}{|c|c|c|}
\hline VARIABLE & FRECUENCIA & Porcentaje \\
\hline Sexo & $\begin{array}{l}\text { Mujer } 57(\mathrm{n}=132) \\
\text { Hombre } 75(\mathrm{n}=132)\end{array}$ & $\begin{array}{l}42.9 \\
56.4\end{array}$ \\
\hline Estrato & $\begin{array}{l}1: 16(n=73) \\
2: 40(n=73) \\
3: 16(n=73) \\
4: 1(n=73)\end{array}$ & $\begin{array}{l}21.91 \\
54.8 \\
21.91 \\
1.4 \\
\end{array}$ \\
\hline Discapacidad & $\begin{array}{c}\text { Discapacidad Intelectual Leve: } 82(\mathrm{n}=127) \\
\text { Discapacidad Intelectual Moderada: } 45(\mathrm{n}=127)\end{array}$ & $\begin{array}{l}64.56 \\
35.43 \\
\end{array}$ \\
\hline Escolaridad acudiente & $\begin{array}{c}\text { No instrucción formal: } 2(\mathrm{n}=33) \\
\text { No terminó primaria: } 7(\mathrm{n}=33) \\
\text { Terminó primaria: } 9(\mathrm{n}=33) \\
\text { Terminó secundaria: } 13(\mathrm{n}=33) \\
\text { Terminó Universidad: } 2(\mathrm{n}=33)\end{array}$ & $\begin{array}{r}6.06 \\
21.21 \\
27.27 \\
39.39 \\
6.06 \\
\end{array}$ \\
\hline Ingreso de las familias & $\begin{array}{l}\text { Menos de } 1 \text { SMMLV: } 22(\mathrm{n}=29) \\
\text { Entre } 1 \text { a } 2 \text { SMMLV: } 7(\mathrm{n}=29)\end{array}$ & $\begin{array}{l}75.86 \\
24.13\end{array}$ \\
\hline Ocupación & $\begin{array}{c}\text { Ama de casa: } 15(\mathrm{n}=32) \\
\text { Trabajador independiente: } 3(\mathrm{n}=32) \\
\text { Desempleado: } 2(\mathrm{n}=32) \\
\text { Empleado: } 12(\mathrm{n}=32)\end{array}$ & $\begin{array}{r}46.87 \\
9.37 \\
6.25 \\
37.5\end{array}$ \\
\hline
\end{tabular}

Elaboración propia, 2017.

() Ediciones Universidad de Salamanca / CC BY-NC-ND

Siglo Cero, vol. 50 (4), n. ${ }^{\circ}$ 272, 2019, octubre-diciembre, pp. 39-59 
CONDICIÓN FÍSICA RELACIONADA CON LA SALUD Y SITUACIÓN SOCIOECÓNOMICA DE NIÑOS Y JÓVENES

CON DISCAPACIDAD INTELECTUAL DE LOS COLEGIOS DISTRITALES DE LA CIUDAD DE BOGOTÁ DIANA ALEXANDRA CAMARGO ROJAS, EDWIN ARCESIO GÓMEZ SERNA Y PABLO SERGIO MOLINA MURCIA

\begin{tabular}{|c|c|c|c|c|c|c|}
\hline $\begin{array}{c}\text { VARIABLES } \\
\text { CONDICIÓN FÍSICA }\end{array}$ & SEXO & $\mathrm{N}$ & Media & $\begin{array}{c}\text { DESVIACIÓN } \\
\text { TÍP. }\end{array}$ & $\begin{array}{c}\text { ERROR } \\
\text { TÍP. DE LA } \\
\text { MEDIA }\end{array}$ & VALOR P \\
\hline \multirow{2}{*}{ IMC } & MUJER & 53 & 22.8874 & 3.73686 & .51330 & 0.010 \\
\hline & HOMBRE & 67 & 21.1320 & 3.58470 & .43794 & \\
\hline \multirow{2}{*}{ SUMPLIEGUES } & MUJER & 32 & 45.1938 & 8,34737 & 1.47562 & 0.000 \\
\hline & HOMBRE & 33 & 35.0303 & 11.33436 & 1.97306 & \\
\hline \multirow{2}{*}{ PORCGRASA } & MUJER & 37 & 27.0649 & 5.95573 & .97912 & 0.000 \\
\hline & HOMBRE & 40 & 16.2875 & 8.05128 & 1.27302 & \\
\hline \multirow{2}{*}{ SITANDREACH } & MUJER & 47 & 12.9149 & 12.01237 & 1.75218 & 0.611 \\
\hline & HOMBRE & 67 & 13.9104 & 8.80553 & 1.07577 & \\
\hline \multirow{2}{*}{ TRUNKLIFT } & MUJER & 27 & 21.3741 & 9.16081 & 1.76300 & 0.307 \\
\hline & HOMBRE & 31 & 19.0548 & 7.98848 & 1.43477 & \\
\hline
\end{tabular}

Elaboración propia, 2017.

En cuanto a la condición socioeconómica de la población con discapacidad del presente estudio, se observa que la mayoría (54\%) de la población que contestó la encuesta STEPS pertenece al estrato 2; en el nivel de escolaridad la mayoría de padres o acudientes han finalizado primaria o secundaria, son muy pocos $(6.06 \%)$ los que cuentan con formación universitaria. En cuanto al ingreso de las familias y la

\begin{tabular}{|c|c|c|c|c|}
\hline \multicolumn{5}{|c|}{$\begin{array}{l}\text { TABLAs 4-5. Pruebas no paramétricas de U Mann Whitney para variables } \\
\text { con comportamiento no normal }\end{array}$} \\
\hline \multicolumn{5}{|c|}{ RANGOS } \\
\hline & SEXO & $\mathrm{N}$ & Rango promedio & Suma de rangos \\
\hline \multirow{3}{*}{ FPRENSIL } & HOMBRE & 52 & 43.18 & 2245.50 \\
\hline & MUJER & 66 & 72.36 & 4775.50 \\
\hline & Total & 118 & & \\
\hline \multirow{3}{*}{ CURLUP } & HOMBRE & 50 & 46.56 & 2328.00 \\
\hline & MUJER & 73 & 72.58 & 5298.00 \\
\hline & Total & 123 & & \\
\hline \multicolumn{5}{|c|}{ ESTADÍSTICOS DE CONTRASTE } \\
\hline & & & FPRENSIL & CURLUP \\
\hline \multicolumn{3}{|c|}{ U de Mann-Whitney } & 867.500 & 1053.000 \\
\hline \multicolumn{3}{|c|}{ W de Wilcoxon } & 2245.500 & 2328.000 \\
\hline \multicolumn{3}{|c|}{$\mathrm{Z}$} & -4.606 & -3.984 \\
\hline \multicolumn{3}{|c|}{ Sig. asintót. (bilateral) } & .000 & .000 \\
\hline
\end{tabular}

a. Variable de agrupación: SEXO.

Fuente: SPSS 20,1.

(C) Ediciones Universidad de Salamanca / CC BY-NC-ND

Siglo Cero, vol. 50 (4), n. ${ }^{\circ} 272,2019$, octubre-diciembre, pp. 39-59 
ocupación de la persona cabeza de familia, se evidencia que la mayoría cuentan con un ingreso menor al salario mínimo mensual vigente y son amas de casa. Estas características permiten concluir que las familias entrevistadas cuentan con una baja condición socioeconómica, siendo esta analizada a partir del estrato socioeconómico, el ingreso de las familias, el nivel de escolaridad y la ocupación.

Dentro de los hallazgos obtenidos es importante resaltar las diferencias significativas por sexo, lo cual le da un lugar a la variable dentro de los perfiles de condición física para la salud en la población con discapacidad. Es por esto, como se evidencia en la Tabla 3, que se observan diferencias significativas ( $\mathrm{p}=<0.001)$ entre hombres y mujeres en las variables relacionadas con composición corporal. En cualidades como flexibilidad y desempeño muscular, evaluadas, por medio del Sit and reach y el Trunk lift, no se evidenciaron diferencias por sexo (ver Tabla 3 y Figuras 1 y 2), a diferencia de la fuerza muscular en donde las diferencias por sexo fueron significativas $(\mathrm{p}=<$ 0.001) (ver Tablas 4 y 5).

\section{Figura 1. Diferencias entre mediana de sumatoria de pliegues por sexo, 2017}

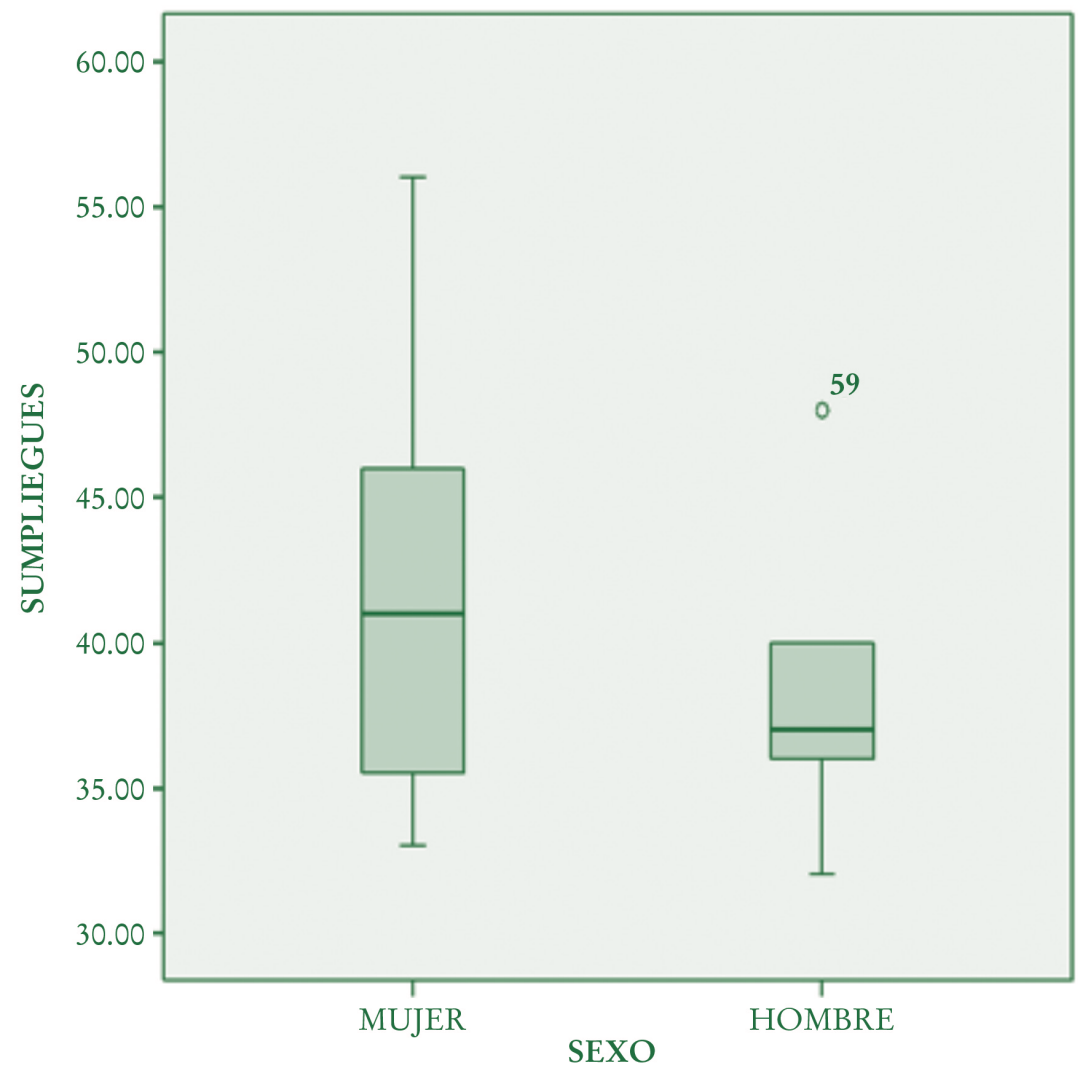

(C) Ediciones Universidad de Salamanca / CC BY-NC-ND

Siglo Cero, vol. 50 (4), n. ${ }^{\circ}$ 272, 2019, octubre-diciembre, pp. 39-59 


\section{Figura 2. Diferencias entre medianas del porcentaje de grasa por sexo, 2017}

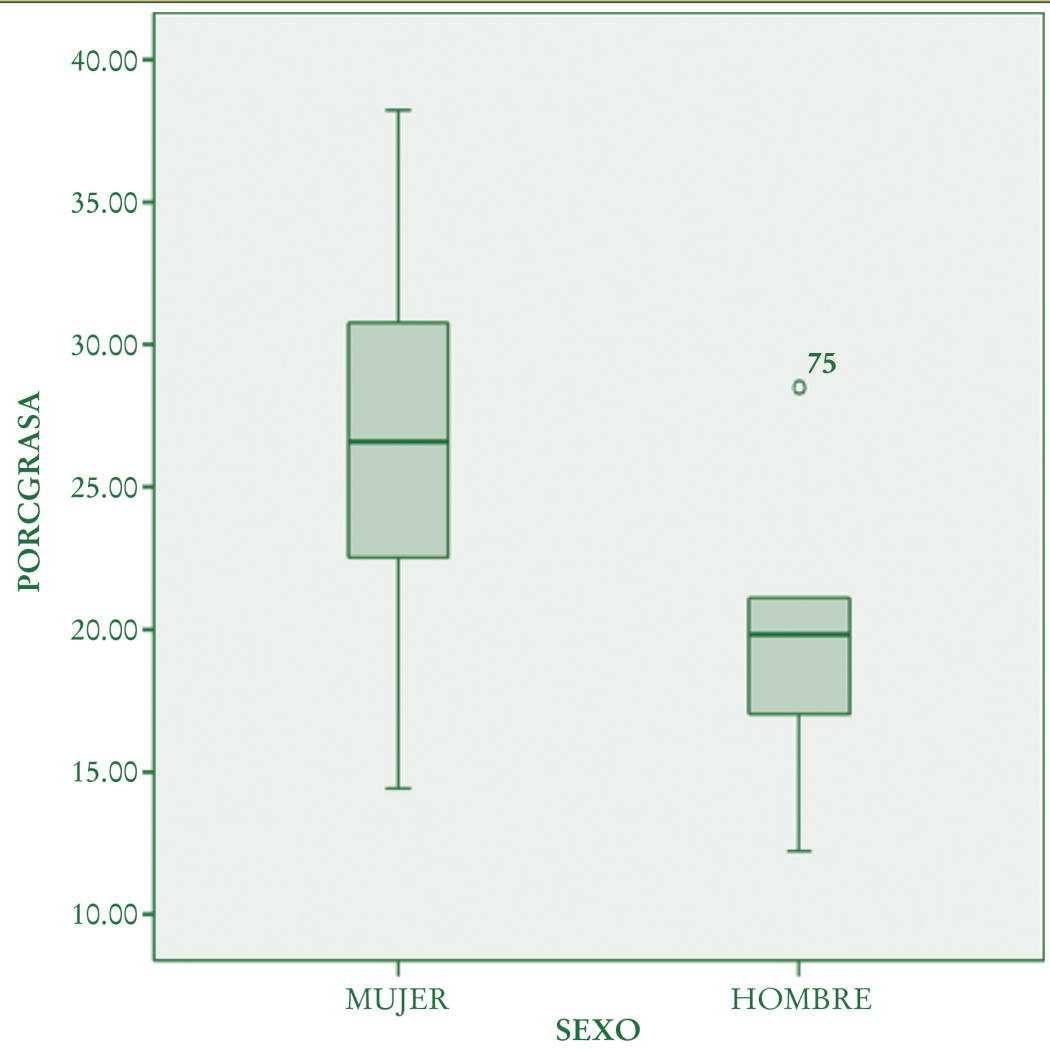

Reconociendo que el tipo de discapacidad puede llegar a influir en el desempeño físico y en la condición física, específicamente en la población con síndrome de Down, en el presente estudio se evidenciaron diferencias significativas en los resultados obtenidos en las pruebas que involucran la flexibilidad, tales como Sit and reach $(\mathrm{p}=0.045)$ y Trunk lift $(\mathrm{p}=0.05)$, lo cual confirma lo expuesto por algunos autores (Tabla 6$)$.

\section{Tabla 6. Prueba T de Student de muestras independientes. Entre diagnóstico,} variables antropométricas y flexibilidad

\begin{tabular}{|c|c|c|c|c|c|c|}
\hline $\begin{array}{c}\text { VARIABLES } \\
\text { CONDICIÓN FÍSICA }\end{array}$ & DISCAPACIDAD & $\mathrm{N}$ & Media & $\begin{array}{l}\text { Desviación } \\
\text { TíP. }\end{array}$ & $\begin{array}{l}\text { ERROR TÍP. } \\
\text { DE LA MEDIA }\end{array}$ & $\begin{array}{l}\text { VALOR } \\
\text { P }\end{array}$ \\
\hline \multirow{2}{*}{ IMC } & $\begin{array}{l}\text { Discapacidad } \\
\text { intelectual moderada }\end{array}$ & 43 & 21.8258 & 3.83109 & .58424 & 0.259 \\
\hline & $\begin{array}{l}\text { Discapacidad leve } \\
\text { síndrome de Down }\end{array}$ & 17 & 23.0109 & 3.02897 & .73463 & \\
\hline
\end{tabular}

(C) Ediciones Universidad de Salamanca / CC BY-NC-ND

Siglo Cero, vol. 50 (4), n. ${ }^{\circ}$ 272, 2019, octubre-diciembre, pp. 39-59 


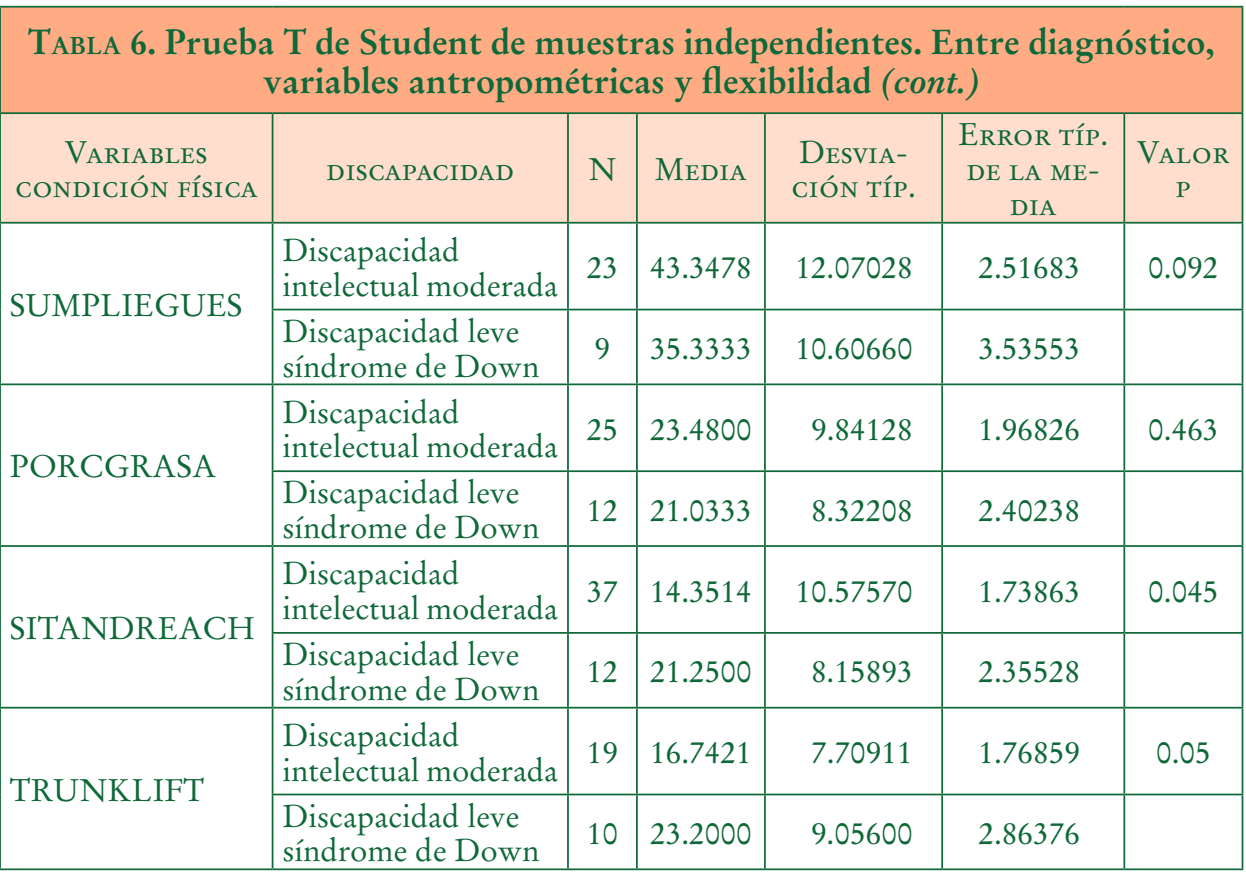

Elaboración propia, 2017.

\subsection{Desenlaces secundarios}

Adicional a los resultados obtenidos que responden a los objetivos de la investigación, se obtuvieron algunos resultados de tipo correlacional que permiten generar unas posibles hipótesis a desarrollar en futuros estudios. Uno de los resultados evidenció una alta correlación entre las pruebas de fuerza muscular, lo cual demuestra que la fuerza prensil y la fuerza abdominal en la población con discapacidad presentan una correlación directamente proporcional con una significancia estadística de $\mathrm{p}=0.001$ (ver Tabla 7).

\begin{tabular}{|c|l|l|c|c|}
\hline \multicolumn{5}{|c|}{ Tabla 7. Correlación de Spearman entre fuerza prensil y Curl up } \\
\hline \multirow{5}{*}{ Rho de Spearman } & & & FPRENSIL & CURLUP \\
\cline { 2 - 5 } & \multirow{3}{*}{ FPRENSIL } & Coeficiente de correlación & 1.000 & $.388(* *)$ \\
\cline { 3 - 5 } & & Sig. (bilateral) & & .000 \\
\cline { 3 - 5 } & \multirow{3}{*}{ CURLUP } & Coeficiente de correlación & $.388(* *)$ & 115 \\
\cline { 3 - 5 } & & Sig. (bilateral) & .000 & 1.000 \\
\cline { 3 - 5 } & $\mathrm{N}$ & 115 & 123 \\
\hline
\end{tabular}

** La correlación es significativa al nivel 0.01 (bilateral).

(C) Ediciones Universidad de Salamanca / CC BY-NC-ND

Siglo Cero, vol. 50 (4), n. ${ }^{\circ}$ 272, 2019, octubre-diciembre, pp. 39-59 
CONDICIÓN FÍSICA RELACIONADA CON LA SALUD Y SITUACIÓN SOCIOECÓNOMICA DE NIÑOS Y JÓVENES CON DISCAPACIDAD INTELECTUAL DE LOS COLEGIOS DISTRITALES DE LA CIUDAD DE BOGOTÁ DIANA ALEXANDRA CAMARGO ROJAS, EDWIN ARCESIO GÓMEZ SERNA Y PABLO SERGIO MOLINA MURCIA

En cuanto a flexibilidad se evidencia dependencia $(\mathrm{p}=0.05)$ entre sexo y las pruebas de flexibilidad de miembro superior, siendo un elemento importante a tener en cuenta dentro de los programas de actividad física dirigidos a la población con discapacidad (ver Tabla 8$)$.

\section{TABLA 8. Chi cuadrado entre sexo y Shoulder strecht Test}

\begin{tabular}{|l|c|c|c|c|c|}
\hline & Valor & GL & $\begin{array}{c}\text { Sig. ASINTÓtica } \\
\text { (BILATERAL) }\end{array}$ & $\begin{array}{c}\text { Sig. EXACTA } \\
\text { (BILATERAL) }\end{array}$ & $\begin{array}{c}\text { Sig. EXACTA } \\
\text { (UNILATERAL) }\end{array}$ \\
\hline Chi-cuadrado de Pearson & $3.825(\mathrm{~b})$ & 1 & .051 & & \\
\hline Corrección por continuidad(a) & 3.123 & 1 & .077 & & \\
\hline Razón de verosimilitudes & 3.838 & 1 & .050 & & \\
\hline Estadístico exacto de Fisher & & & & .061 & .038 \\
\hline Asociación lineal por lineal & 3.792 & 1 & .052 & & \\
\hline N de casos válidos & 116 & & & & \\
\hline
\end{tabular}

a Calculado solo para una tabla de $2 \times 2$.

b 0 casillas $(.0 \%)$ tienen una frecuencia esperada inferior a 5 . La frecuencia mínima esperada es 22.81 .

Por último, es importante señalar que se evidencia una correlación inversamente proporcional entre la sumatoria de pliegues y la fuerza prensil $(r=-0.366 ; p=0.01)$, con una alta significancia estadística, lo cual se corresponde con los hallazgos de poblaciones sin discapacidad, convirtiendo la fuerza prensil en un posible predictor de indicadores relacionados indirectamente con el riesgo cardiovascular y la probabilidad de presentar enfermedades no transmisibles. Así mismo, se encontró una correlación positiva $(r=0.449 ; \mathrm{p}=0.01)$ entre la prueba de curl up y porcentaje de grasa, siendo estas dos variables componentes a tener en cuenta dentro de la valoración de condición física para la salud (ver Tabla 9).

\begin{tabular}{|c|c|c|c|c|c|c|c|c|c|c|}
\hline \multicolumn{11}{|c|}{$\begin{array}{c}\text { TABLA 9. Correlación de Spearman entre cualidades físicas y condiciones } \\
\text { antropométricas }\end{array}$} \\
\hline & & & $\begin{array}{l}\text { 最 } \\
\text { 覓 } \\
\text { 空 }\end{array}$ & 含 & 是 & $\sum_{i}^{U}$ & $\begin{array}{l}\text { 留 } \\
\text { 恶 } \\
\text { 点 }\end{array}$ & 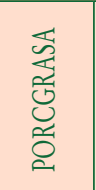 & 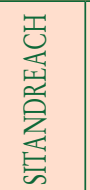 & 䛒 \\
\hline \multirow{6}{*}{ 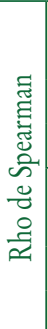 } & \multirow[t]{3}{*}{ FPRENSIL } & $\begin{array}{l}\text { Coeficiente de } \\
\text { correlación }\end{array}$ & 1.000 & $.388(* * *)$ & -.081 & -.155 & $-386(* * *)$ & $-355(* * *)$ & .157 & .024 \\
\hline & & Sig. (bilateral) & & .000 & .380 & .094 & .002 & .002 & .109 & .858 \\
\hline & & $\mathrm{N}$ & 118 & 115 & 118 & 118 & 64 & 75 & 106 & 58 \\
\hline & \multirow[t]{3}{*}{\begin{tabular}{|l|} 
CURLUP \\
\end{tabular}} & $\begin{array}{l}\text { Coeficiente de } \\
\text { correlación }\end{array}$ & $.388(* * *)$ & 1.000 & $-389\left(^{(* *)}\right.$ & $-.327(* * *)$ & -190 & $-.449(* * *)$ & $.299(* * *)$ & -.083 \\
\hline & & Sig. (bilateral) & .000 & & .000 & .000 & .139 & .000 & .001 & .533 \\
\hline & & $\mathrm{N}$ & 115 & 123 & 123 & 116 & 62 & 73 & 113 & 58 \\
\hline
\end{tabular}

(C) Ediciones Universidad de Salamanca / CC BY-NC-ND

Siglo Cero, vol. 50 (4), n. ${ }^{\circ}$ 272, 2019, octubre-diciembre, pp. 39-59 


\section{TABLA 9. Correlación de Spearman entre cualidades físicas y condiciones antropométricas (cont.)}

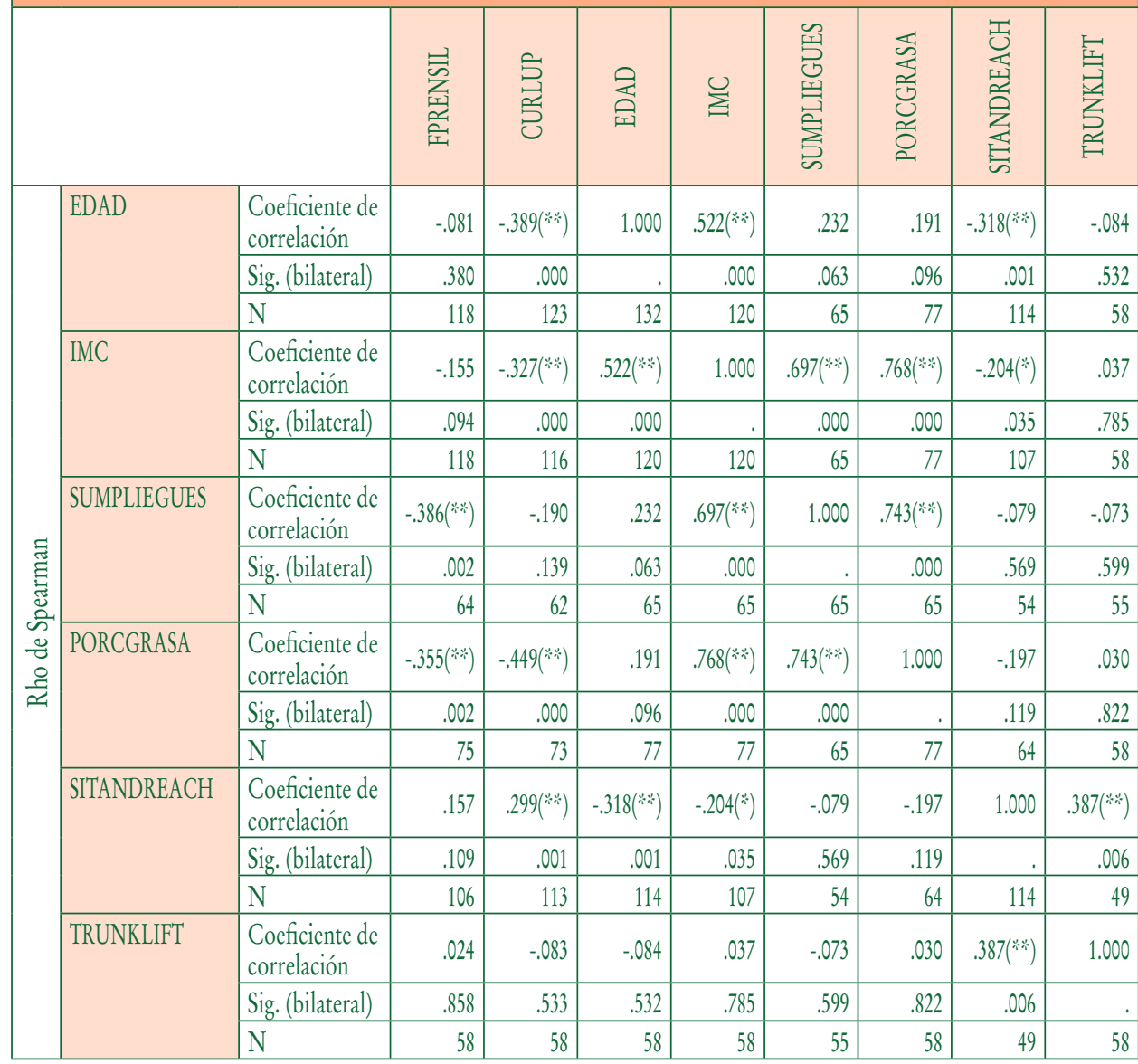

* La correlación es significativa al nivel 0.05 (bilateral).

* La correlación es significativa al nivel 0.01 (bilateral).

\section{Discusión}

En el presente estudio, con relación al género y la discapacidad, se evidencia un mayor número de hombres con discapacidad $(56.81 \%$; 75$)$ en comparación a las mujeres $(43.18 \%$; 57$)$; comportamiento similar al de la población bogotana, lo que concuerda con los estudios sobre la población con discapacidad en Bogotá (DANE, 2010), en donde de un total de 18084 niños y jóvenes con discapacidad de 10 a 18 años el 55\% (99499) son hombres y el $44.9 \%$ (8135) son mujeres. 
CONDICIÓN FÍSICA RELACIONADA CON LA SALUD Y SITUACIÓN SOCIOECÓNOMICA DE NIÑOS Y JÓVENES CON DISCAPACIDAD INTELECTUAL DE LOS COLEGIOS DISTRITALES DE LA CIUDAD DE BOGOTÁ

DIANA ALEXANDRA CAMARGO ROJAS, EDWIN ARCESIO GÓMEZ SERNA Y PABLO SERGIO MOLINA MURCIA

La condición socioeconómica de las familias es una variable que se aproxima al estudio de la exclusión social de la población con discapacidad (Cruz y Hernández, 2008). El presente estudio evidencia que la mayoría de la población se encuentra en el estrato 2, y con un ingreso familiar de menos de un salario mínimo; a esto se suma que la mayoría de los acudientes no han finalizado la primaria o no han recibido una instrucción formal por lo que su ocupación es de amas de casa o trabajadores informales. Esta condición se evidencia igualmente en el estudio realizado por Hernández y Hernández (2005) citado por Cruz y Hernández (2008), en el cual se analizó el registro de los hogares de personas con discapacidad encontrando dificultades en el campo laboral.

La condición socioeconómica en diferentes estudios se asocia con los modos de vida de las familias, los cuales a su vez inciden o influyen en el estilo de vida y las condiciones de salud de niños y jóvenes con discapacidad. Este modo de vida definido desde el modelo de determinación social como "la realidad histórica a la que pertenece un grupo social determinado" (Breilh, 2003; citado por Camargo y Mena, 2009: 19) incluye las redes de apoyo y las condiciones socioeconómicas de las familias; elementos referenciados por Montenegro y Camargo (2015) como determinantes de la práctica de la actividad física en la población.

Uno de los elementos a tener en cuenta dentro de los perfiles epidemiológicos, relacionados con las enfermedades crónicas no transmisibles, es la condición física para la salud, definida por el American College of Sports Medicine (ACSM) como determinante de la actitud física y del riesgo cardiovascular. Esta condición en la población con discapacidad intelectual del presente estudio evidenció resultados similares a los resultados del estudio de Bofill (2008), tales como un mayor porcentaje de grasa, sumatoria de pliegues corporales e índice de masa corporal en las niñas, en comparación a los niños con este tipo de discapacidad $(\mathrm{p}=0.000)$. Este mismo comportamiento se evidenció en las variables de fuerza muscular.

Dentro de los estudios realizados en la población con discapacidad se sugiere hacer diferencias por tipo de diagnóstico (Jin-Ding et al., 2010). En el presente estudio las diferencias entre retraso mental leve y moderado no son significativas para ninguna de las variables de estudio. Por otro lado, las diferencias entre síndrome de Down y retraso mental solamente se evidenciaron en las pruebas de flexibilidad "Sit and reach" y "Trunk lift", las cuales evalúan las cadenas musculares anteroposteriores de tronco y miembros inferiores.

La mayoría de la población (69\%) respecto a la sumatoria de pliegues, presenta una condición no saludable determinada a partir de baremos validados para la población con discapacidad intelectual. No obstante, este resultado presenta diferencias con los obtenidos en porcentaje de grasa, donde el $20 \%$ de la población presenta sobrepeso y obesidad como riesgo para su salud. Esto sugiere la revisión de la confiabilidad de la prueba relacionada con la toma de pliegues corporales ya que requiere del entrenamiento o de la experticia de los evaluadores, lo cual es objeto de estudio de la segunda fase de la presente investigación.

Las condiciones de fuerza y flexibilidad siguen siendo cualidades físicas para la salud a trabajar en la población, las cuales no están determinadas por el diagnostico,

(C) Ediciones Universidad de Salamanca / CC BY-NC-ND

Siglo Cero, vol. 50 (4), n. ${ }^{\circ}$ 272, 2019, octubre-diciembre, pp. 39-59 
CONDICIÓN FÍSICA RELACIONADA CON LA SALUD Y SITUACIÓN SOCIOECÓNOMICA DE NIÑOS Y JÓVENES CON DISCAPACIDAD INTELECTUAL DE LOS COLEGIOS DISTRITALES DE LA CIUDAD DE BOGOTÁ

DIANA ALEXANDRA CAMARGO ROJAS, EDWIN ARCESIO GÓMEZ SERNA Y PABLO SERGIO MOLINA MURCIA

debido a que fueron evaluadas con parámetros establecidos para la población con discapacidad, lo que nos permite afirmar que en la población evaluada se evidencia una baja condición física posiblemente asociada al sedentarismo.

Por último, frente a los resultados secundarios, se observó en el presente estudio correlación inversamente proporcional entre fuerza prensil e indicadores indirectos de riesgo cardiovascular como la sumatoria de pliegues $(r=-0.366 ; p=0.01)$, como se evidenció en el estudio de Triana y Ramírez (2013), en donde encontraron "correlaciones inversas ... entre la fuerza muscular y los indicadores de adiposidad $(r=-0.317$; $\mathrm{p}=0.001)$, circunferencia de la cintura $(\mathrm{r}=-0.309 ; \mathrm{p}=0.001)$ ” (p. 433).

Es importante mencionar que el presente estudio se enfocó en la caracterización de la condición física y la condición socioeconómica de la población con discapacidad intelectual; no obstante, al encontrar índices de sobrepeso y obesidad, así como una baja condición de fuerza y resistencia cardiovascular, se considera que es una población con alto riesgo cardiovascular, el cual puede estar asociado a la inactividad física de la población, que en su mayoría depende de la existencia de facilitadores que favorezcan el acceso a la práctica de la actividad física.

Frente a esto, Carbó, Guàrdia, Giné y Schalock (2016) plantean que los efectos de la práctica de actividad física en la condición física de personas con DI han sido ampliamente documentados, principalmente en la resistencia cardiovascular (Rimmer, Heller, Wang y Valerio, 2004, citado por Carbó et al., 2016), en la fuerza muscular y en el equilibrio (Carmeli et al., 2005; Shields et al., 2013, citados por Carbó et al., 2016). No obstante, según Carbó et al. (2016), "algunos artículos señalan que los individuos con DI muestran bajos niveles de AF, insuficientes para alcanzar los niveles de salud esperados" (Fernhall y Pitetti, 2001; Temple, Frey y Stanish, 2006; citados por Carbó et al., 2016, pp. 149).

\section{Conclusiones}

La condición física y la probabilidad de presentar enfermedades no transmisibles no están relacionadas con la presencia de discapacidad intelectual (con o sin síndrome de Down para el presente estudio), como lo plantean algunos estudios, sino con diferentes factores influyentes que se encuentran en el modo y estilo de vida de los sujetos; estas relaciones son similares a las que se dan en la población que no presenta ningún tipo de discapacidad. Sin embargo, condiciones sociales, estructurales y políticas pueden generan que en la población con discapacidad la magnitud del problema aumente por la falta de escenarios y/o programas inclusivos que incentiven la práctica de actividad física, así como la presencia de imaginarios sociales de la discapacidad cercanos a la enfermedad y la deficiencia, lo que lleva a que las instituciones educativas no contemplen en su currículo, desde la clase de educación física, la promoción de la actividad física en este tipo de población; su énfasis curricular está en los procesos cognitivos y sus avances y no en la práctica de la actividad física. Por lo anterior, conocer las condiciones de la población, sus características reales y capacidades permite generar programas dirigidos a la población que continúen potencializando su desarrollo humano y social.

(C) Ediciones Universidad de Salamanca / CC BY-NC-ND

Siglo Cero, vol. 50 (4), n. ${ }^{\circ}$ 272, 2019, octubre-diciembre, pp. 39-59 
CONDICIÓN FÍSICA RELACIONADA CON LA SALUD Y SITUACIÓN SOCIOECÓNOMICA DE NIÑOS Y JÓVENES CON DISCAPACIDAD INTELECTUAL DE LOS COLEGIOS DISTRITALES DE LA CIUDAD DE BOGOTÁ

DIANA ALEXANDRA CAMARGO ROJAS, EDWIN ARCESIO GÓMEZ SERNA Y PABLO SERGIO MOLINA MURCIA

\section{Referencias bibliográficas}

AAIDD (2010). Intellectual disability. Definition, classification, and systems of supports. Washington, D.C.: American Association on Intellectual and Developmental Disabilities [Traducción al castellano: AAIDD (2011). Discapacidad intelectual. Definición, clasificación y sistemas de apoyo. Madrid: Alianza Editorial].

American College of Sports Medicine - ACSM (2009). ACSM'S Exercise management for persons with chronic disease and disabilities. Third Edition. Champaing, Illinois: Human Kinetics.

American College of Sports Medicine - ACSM (2014). Guidelines for exercise testing and prescription. Nine Edition. Baltimore, Philadelphia: Wolters Kluwer Health.

Bofill, A. (2008). Valoración de la condición física en la discapacidad intelectual. Tesis doctoral. Programa de Doctorado: Diferencias Educativas e Igualdad de Oportunidades. Departamento de Obstetricia y Ginecología, Pediatría, Radiología y Anatomía. Universidad de Barcelona. España.

Buhring, B. y Oliva, M. (2009). Determinación no experimental de la conducta sedentaria en escolares. Revista Chilena de Nutrición, 36 (1), 23-30.

Camargo, D. y Mena, B. (2009). Análisis de la condición de salud de los usuarios de Unisalud con osteoartrosis de rodilla, desde el modelo de la determinación social de la epidemiología crítica. Tesis para optar al título de Magíster en Salud pública. Universidad Nacional de Colombia. Bogotá, Colombia.

Carbó, M., Guàrdia, J., Giné, C. y Schalock, R. (2016). A structural equation model of the relationship between physical activity and quality of life. International Journal of Clinical and Health Psychology, 16, 147-156.

Casimiro, A. (2002). Hábitos deportivos y los estilos de vida de los escolares almerienses. Almería: Universidad de Almería.

Congreso de la República (1994). Ley 115 de Educación Nacional.

Contreras, J. M. (2009). Didáctica de la educación física. Barcelona: INDE.

Cossío, M. y Bolaños, A. (2015). Perfil antropométrico en función del estado nutricional de niños con discapacidad intelectual. Revista Chilena de Pediatría, 86 (1), 18-24.

Cruz, I. y Hernández, J. (2008). Magnitud de la discapacidad en Colombia: una aproximación a sus determinantes. Revista de Ciencias de la Salud, 6 (3), 23-35.

DANE (2010). Trabajo orientado para implementar en todo el país el registro continuo para la localización y caracterización de las personas con discapacidad. Consultado en el 2017 en: www.dande.gov.co/index.php/estadísticas-guiontema/salud/discapacidad.

FERnández, J. y Hoyos, L. (2007). Perfil de las cualidades físicas y antropométricas de los escolares colombianos. Bogotá: Universidad Pedagógica Nacional.

Foley, J. T., Harvey, S., Hae-Ja, C. y So-Yeun, K. (2008). The relationships among fundamental motor skill, health-related physical fitness, and body fatness in south Korean adolescents with mental retardation. Research Quarterly for Exercise and Sport, 2, 149-157.

Jin-Ding, L., Pei-Ying, L., Lan-Ping L., Yu-Yu, C., Sheng-Ru, W. y Jia-Ling, W. (2010). Physical activity and its determinants among adolescents with intellectual disabilities. Research in Developmental Disabilities, 31, 263-269.

Kleinert, H., Miracle, S. y Sheppard-Jones, K. (2007). Including students with moderate and severe intellectual disabilities in school extracurricular and community recreation activities. Intellectual and Developmental Disabilities, 45 (1), 46-55. 
CONDICIÓN FÍSICA RELACIONADA CON LA SALUD Y SITUACIÓN SOCIOECÓNOMICA DE NIÑOS Y JÓVENES CON DISCAPACIDAD INTELECTUAL DE LOS COLEGIOS DISTRITALES DE LA CIUDAD DE BOGOTÁ

DIANA ALEXANDRA CAMARGO ROJAS, EDWIN ARCESIO GÓMEZ SERNA Y PABLO SERGIO MOLINA MURCIA

Krebs, P. (2000). Adapted physical education and Sport. Champaign, IL: Human Kinetics. Recuperado el 14 de marzo de 2010 en la base de datos de Academic Search Complete.

Krieger, N. (2002). Glosario de epidemiología social. Revista Panameña de Salud Públical Pan Am J Public Health, 11 (5/6), 480-490.

Li, H., Frey, G., McCormick, B. y Johnston, J. (2015). Comparison of obesity among Chinese and U.S. Special Olympic athletes with intellectual disabilities. Research in Developmental Disabilities, 41-42, 94-100.

Lopategui, E. (2008). Determinación de la razón cintura cadera. Recuperado en junio 2016 en: http://www.saludmed.com/LabFisio/Lab-F-Men1.html.

MEN (2007). Educación para todos. Altablero, 43. Mayor información: http://www.mineducacion.gov.co/1621/article-141881.html.

Montenegro, M. y Camargo, D. A. (2015). Abordajes teóricos en la comprensión de la influencia de los recursos económicos en la actividad física. Tesis maestría de Actividad física para la salud. Universidad del Rosario. Bogotá, Colombia.

Moreno, M (2007). Políticas y concepciones en discapacidad: un binomio por explorar. Universidad Nacional de Colombia. Facultad de Medicina. Doctorado en Salud Pública. Bogotá, Colombia. Recuperado en diciembre del 2015 en: http://www.bdigital.unal.edu. co/4586/1/9789587017762.pdf.

Ortiz, E. (2010). Estado de la situación de la aptitud física en niños y niñas con discapacidad intelectual en las edades escolares. Sistema Universitario Ana G. Méndez Universidad Metropolitana. Recinto Cupey. Escuela Graduada de Educación. Recuperado el 13 de febrero de 2016 de http://www.suagm.edu/umet/biblioteca/UMTESIS/Tesis_Educacion/ Ens_Educ_Fisica/ELaureano_122010.pdf.

SHIN, I. y PARK, E. (2012). Meta-analysis of the effect of exercise programs for individuals with intellectual disabilities. Research in Developmental Disabilities, 33, 1937-1947.

Triana, H. y Ramírez, R. (2013). Asociación de la fuerza muscular con marcadores tempranos de riesgo cardiovascular en adultos sedentarios. Endocrinología y Nutrición, 60 (8), 433438. doi: 10.1016/j.endonu.2013.01.009.

Vidarte, J., Vélez, C. y Parra, J. (2012). Niveles de sedentarismo en población de 18 a 60 años. Manizales, Colombia. Revista de Salud Pública, 14 (3), 417-428.

Villarreal, J. (2011). Diseño de una propuesta de planeación, puesta en práctica y evaluación en matemáticas para aulas inclusivas. Recuperado el 19 de octubre de 2015 de http://funes. uniandes.edu.co/1848/1/50476285-DISENO-DE-UNA-PROPUESTA-DE-PLANEACION-PUESTA-EN-PRACTICA-Y-EVALUACION-EN-MATEMATICAS-PARAAULAS-INCLUSIVAS-PONENCIA.pdf.

Winnick, J. P. y Short, F. X. (1999). The Brockport Physical Fitness Test Manual. A Health - Related Test for Youngsters with Physical and Mental Disabilities. Champaign, Illinois: Human Kinetics.

Winnick, J. P. y Short, F. X. (2014). The Brockport Physical Fitness Test Manual. A HealthRelated Test for Youngsters with Physical and Mental Disabilities. Second Edition. Champaign, Illinois: Human Kinetics. 


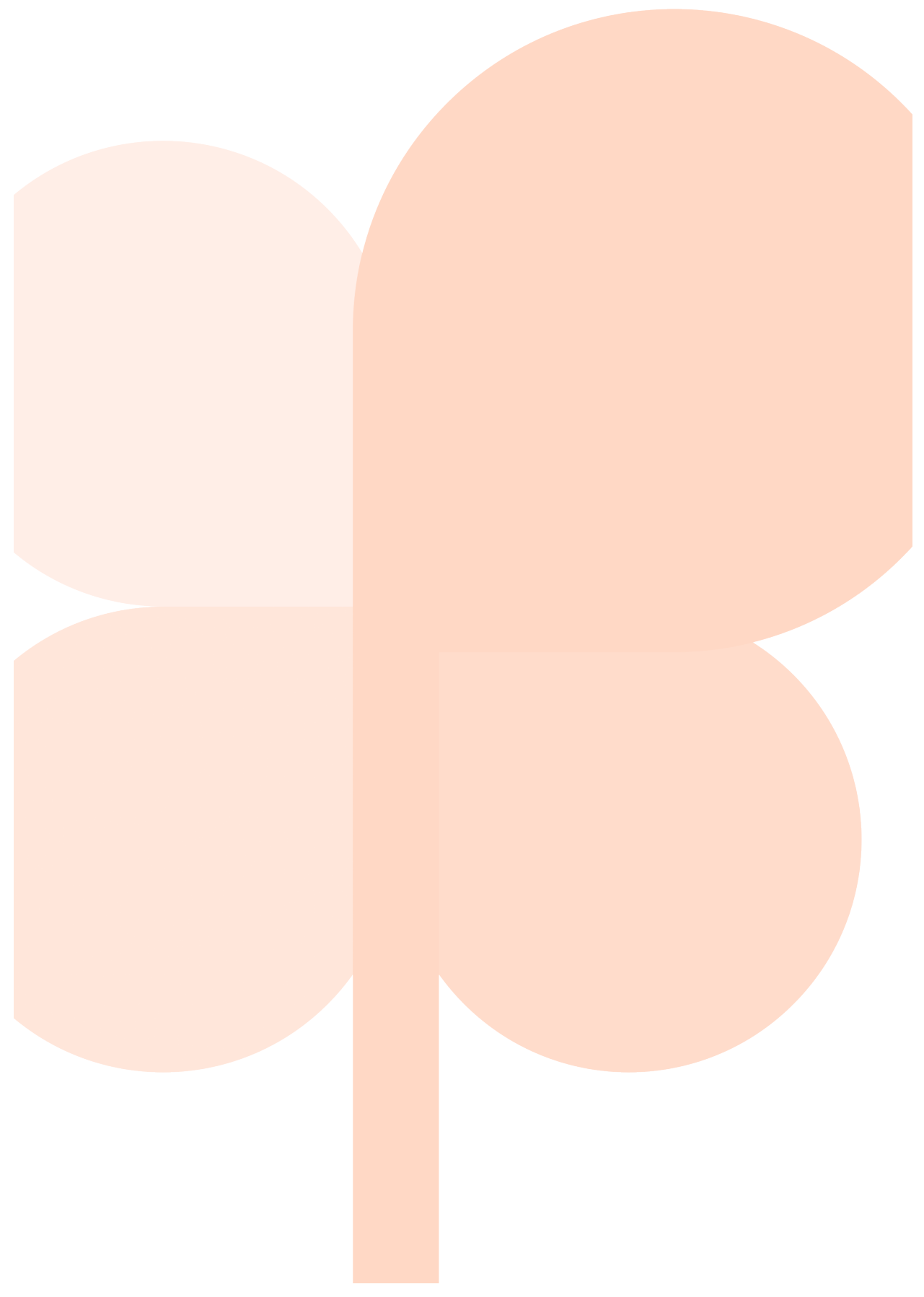

